\title{
An Analysis of Recipe-based Instruction in an Introductory Fluid Mechanics Laboratory
}

\section{Dr. Blake Everett Johnson, University of Illinois at Urbana-Champaign}

Dr. Blake Everett Johnson received his doctorate in Theoretical and Applied Mechanics at the University of Illinois at Urbana-Champaign in 2012 for his experimental studies of heated jets in cross-flow. Other topics of interest to him include boundary layer turbulence over realistic rough surfaces, film cooling of gas turbine engines, pressure-sensitive paint, and development of new teaching methods in introductory mechanics courses. Dr. Johnson now works as a lecturer and lab manager in the Department of Mechanical Science and Engineering (MechSE) at the University of Illinois. While remaining interested and active in the field of experimental fluid mechanics, he has chosen to spend most of his professional energy on improving the teaching of thermo/fluids laboratory courses through the development of engaging and intellectually-stimulating laboratory exercises, as well as improving introductory mechanics education and design courses in the MechSE department.

Jason W Morphew, University of Illinois at Urbana-Champaign 


\title{
An Analysis of Recipe-Based Instruction in an Introductory Fluid Mechanics Laboratory
}

\author{
Blake Everett Johnson and Jason Morphew \\ University of Illinois at Urbana-Champaign
}

\begin{abstract}
Undergraduate mechanics laboratory classes have frequently been taught with a primary goal of demonstrating principles successfully through experimentation and a secondary goal of teaching proper experimental techniques, among others. Historically, this set of priorities has motivated laboratory courses to provide students with prescribed experimental methods, i.e. a "recipe" to follow. Because mechanics principles are already taught in the associated lecture portion of our mechanics classes, demonstration of them in the laboratory may be considered redundant. Research has demonstrated that labs which encourage active learning and inquiry promote the development of higher order skills and may lead to better preparation for future learning. This paper describes an intervention where lab sections were randomly assigned to either receive a recipe or to follow their own laboratory procedures. Results indicate that students who were required to utilize their own laboratory procedures outperformed students who received a recipe on their lab reports. Educational implications for these findings are discussed.
\end{abstract}

\section{Keywords}

Inquiry-based instruction, laboratory course, fluid mechanics

\section{Introduction}

We report on a preliminary study where the level of inquiry in an introductory fluid mechanics laboratory course seemed to affect student engagement in the lab exercises. In this study, we describe a shift in laboratory procedures from following of rote procedures, a so-called "recipe"based approach, to one where students are given freedom to design and implement their own procedures, an inquiry-based approach. This study is timely in that it occurred at a time when there is a push in engineering education toward inquiry-based learning, as described by Froyd, et al. ${ }^{1}$, who explain that inquiry-based learning exercises comprise part of a major shift in current engineering education trends, whereby the results of education research are being used to modify engineering curricula. A study by Self and Widmann ${ }^{2}$ demonstrated how inquiry-based learning activities show promise for teaching non-intuitive mechanics principles and convincing students to trust the scientific theories rather than their own faulty intuition. Froyd, et al. ${ }^{1}$ mentions an additional evidence-based trend in engineering education where environments are created encouraging active engagement of students during learning exercises. Engineering students rarely 
begin their curricula highly motivated to do engineering, but can graduate highly motivated if faculty make a point of emphasis to engage their learning along the way. A similar observation was made by Regueras, et al. ${ }^{3}$, who investigated different approaches for motivating students' learning in collaborative and competitive environments, showing that the students enjoyed these particular learning environments more than the traditional approach taken in their other courses, where no such learning environments were intentionally implemented. Observations were made by Garrison, et al. ${ }^{4}$ of the effects of a guide document being provided to help high school students learn how to design experiments. This study, though it differs from the one we have conducted in that a vastly different set of students was analyzed, suggests that a guiding document aided in the ability of students to learn how to design an experiment.

In engineering research and industrial practice, live troubleshooting is an important aspect of critical thinking that, when practiced habitually, may save great amounts of time and resources. Unfortunately, live troubleshooting is a habit that is frequently under-emphasized or ignored entirely in undergraduate engineering education. Many career engineers do not develop a tendency to live-test their measured values until after they have experienced some failures that would otherwise have been preventable had they checked values for reasonability during data acquisition. In addition, industry professionals have identified troubleshooting as a key educational outcome for engineering students, as noted by Heer, et al. ${ }^{5}$ Following informal observations in our laboratory courses, we suspect that the prevalence of "black box" measurement devices, which receive analog physical inputs and report a measured value digitally, contributes to this problem. These devices are appealing to engineers because of their ease of use and the immediacy with which they provide measurements in desired units. However, naïve engineering students, eager for simplicity and not knowing how they should be properly operated, tend to put too much trust in such devices without realizing that they require precise calibration, the accuracy of which may degrade over time, and proper usage. This trust often results in students believing that these devices give them the one "true" value of a quantity, without doing any sort of check to confirm the reliability of the measurement. When students trust measurements as recording the "true" value, they tend to focus on following procedures and memorization rather than interpretation and explanation during laboratory investigations ${ }^{6}$. For introductory-level students, it may be too much to ask them to understand the full analog-to-digital conversion process that a measurement device does, and our course does not seek to teach such things. However, it can be considered within the scope of our class for the students to offer some justification for what degree to which their measurements are to be trusted.

Prior to our study, the lab course in question had for many years utilized a recipe-based model to guiding students through the experimental procedures in their lab course. However, prompted by review of ABET outcomes in 2013, faculty in the Mechanical Science and Engineering department at the University of Illinois decided that the lab course was failing at teaching students how to design experiments.

A new approach was then adopted whereby students would, before conducting their experiments, practice designing the experiment themselves. They were required to analyze the background 
theory, learn how the measurement equipment works, and then identify the dependent, independent, and control variables before designing their own step-by-step experimental procedure to follow for testing the theory. Their handwritten experiment designs are then turned in and graded, satisfying the ABET requirement. Grading is done based on the categorization of variables and the completeness and usefulness of the procedures that they prepare.

After preparing an experiment design, students would nonetheless be given a recipe of experimental procedures to use for conducting the actual experiment. Continuation of this recipebased approach was motivated by a concern that they might not conduct their experiment properly and that the students then might not trust the concepts that they were learning in the lecture portion of the course. Informal observations of student behavior during this period indicated that few students seemed to be interested in the work that they were doing in the lab. In a typical group of four students who were supposed to be working together to perform an experiment, one or two students would do most of the work while the others sat passively by, uninvolved and disinterested.

Then, for one semester, a new approach was adopted where none of the students would be given a recipe and would be expected to follow their own experiment procedures. Teaching assistants (TAs) were instructed not to correct the students' procedures unless they were deemed at risk to damage equipment, and they would not interfere with the design of experiment unless requested to help, in which event they would offer advice leading to a proper change in the experiment, but never recommending specific changes. Informal observations made by the TAs suggested that there was widespread improvement in the students' experiences in the lab. Students were rarely observed not to participate anymore, lively discussions involving all team members became frequent, and students who were unable to collect data nonetheless participated by livetroubleshooting the data. That is, using their calculators or laptops to process the raw data and confirm whether it seemed reasonable even though they were never prompted to do so.

This study is being conducted to report the observed differences in student participation and involvement when the recipe is given or withheld, as determined by TA observation. Additionally, long-term effects of recipe-based direction are analyzed, including whether there is an observable effect on future learning, experimentation habits such as live troubleshooting, group dynamics, and individual grades.

\section{Study Design}

\section{Description of the Two Lab Conditions}

Lab sections were divided into two conditions: those that would receive a recipe and those that would not. Lab sections that met on Mondays, Wednesdays, and Fridays were given a folder that contained the recipe after completion of their design of experiment exercise. A sample of the recipe for one of the lab exercises is shown in Figure 1. Lab sections that met on Tuesdays and Thursdays were not given the recipe and the students were instructed to use their own experimental procedures once they completed them, as shown in Table 1. Seven lab TAs led the lab sections, 
Procedure:

1. Open the FM50 Centrifugal Pump Software. It should look like Figure 9.

2. Set the desired pump setting speed.

3. Make sure the flow control valve is fully open.

4. Click the on/off switch to show a "1"

5. Wait a few minutes for the pump to reach steady state.

6. Make sure you have an empty table for the data. If not, click the icon for a nev

7. Once steady state is reached, click the record data button. This may be do (control or table).

8. Close the valve ONE QUARTER TURN.

9. Wait a minute to establish steady state again. Then click "record data".

10. Repeat steps 8 and 9 until the valve is fully closed and there is no flow rate.

11. After the last data point is taken at zero flow rate, fully open the valve for max

12 Return to the control diacram if needed and chance the numn cettina cnead

Figure 1. A sample experimental procedures recipe for M/W/F students to follow.

five of whom led multiple lab sections. All TAs whose appointments required them to teach two or more lab sections were assigned to teach both types of laboratory section (i.e. at least one each of a M/W/F lab and a Tu/Th lab).

Students were all required to review the laboratory manual prior to coming to each lab session, which was enforced by the possibility of the TA formally quizzing them on the content of the manual. This practice was used during the lab exercise prior to this study to encourage the students to take seriously the need to review the manual. Lab manuals contain all of the background theory and a description of the experimental equipment, which the TAs also review at the beginning of the lab sessions. All students are taught about usage of all equipment and given freedom to "tinker" with the equipment in order to understand how it works prior to and during the experiment.

Each regular laboratory exercise in the course takes two weeks to complete. This study was conducted over five weeks and covered the second and third laboratory exercises of the semester (Lab 2 and Lab 3, respectively). In addition, a one-week-long Lab Practical exercise (Lab 3b) was performed after Lab 3, as shown in Table 2. The remaining lab exercises (Labs 1, 4, and a fourweek-long design project) were conducted with no differences in instruction and were not used as a part of this study. 
Table 1. Laboratory agenda for M, W, F lab sections and Tu, Th lab sections.

\begin{tabular}{c|c|c} 
Lab Minutes & M, W, \& F Lab Section Activities & Tu \& Th Lab Section Activities \\
\hline \hline Week 1: 0-15 & TA gives introductory lecturette & TA gives introductory lecturette \\
\hline Week 1: 15-45 & $\begin{array}{c}\text { Students work in groups to prepare } \\
\text { their design of experiment }\end{array}$ & $\begin{array}{c}\text { Students work in groups to prepare } \\
\text { their design of experiment }\end{array}$ \\
\hline Week 1: 45-60 & $\begin{array}{c}\text { TAs make copies of designs of } \\
\text { experiment for plagiarism checking }\end{array}$ & $\begin{array}{c}\text { TAs make copies of designs of } \\
\text { experiment for plagiarism checking }\end{array}$ \\
\hline Week 1: 60-120 & $\begin{array}{c}\text { TAs give folder containing recipe, } \\
\text { students begin performing } \\
\text { experiment }\end{array}$ & $\begin{array}{c}\text { Students begin performing } \\
\text { experiment, following their own } \\
\text { procedures }\end{array}$ \\
\hline Week 2: 0-120 & $\begin{array}{c}\text { Students given recipe folder, finish } \\
\text { performing experiment }\end{array}$ & $\begin{array}{c}\text { Students finish performing } \\
\text { experiment, following their own } \\
\text { procedures }\end{array}$ \\
& &
\end{tabular}

Description of Labs 2 and 3

Lab 2 is an experiment where students investigate the performance of centrifugal pumps, including relationships between pump head and efficiency vs flowrate at varying degrees of input power to the pump. All of the equipment is fully contained in a tabletop setup where a pump circulates water from a reservoir through a series of valves and measurement devices. A PC is used to control the power to the pump and samples the water temperature, flowrate, pump speed, and the pressure

Table 2. Laboratory course semester schedule

\begin{tabular}{c|c|c} 
Lab Activity & Duration & Part of Study \\
\hline \hline Lab 1: Basic Fluid Properties & 2 weeks & No \\
\hline Lab 2: Centrifugal Pump Investigation & 2 weeks & Yes \\
\hline Lab 3: Free Jet Investigation & 2 weeks & Yes \\
\hline Lab 3b: Free Jet Challenge & 1 week & Yes \\
\hline Lab 4: Wake Behind a Cylinder & 2 weeks & No \\
\hline Design Project: System of Fountains & 4 weeks & No
\end{tabular}


rise across the pump. The raw data is saved in an Excel spreadsheet for the students to process and analyze. Students control experimental parameters such as the power, which is controlled digitally by entering a percentage of the maximum power for the software to run at, and the flowrate, which is controlled by manual adjustment of a globe valve to change the pressure drop through the pump. All of the sensors are electronic and the students are never taught how they work but assumed to trust that they report accurate readings. Nonetheless, the data can be used to generate performance curves that can be compared to published curves for centrifugal pumps.

Lab 3 is an experiment where students measure the velocity profile of an incompressible free jet through the use of a Pitot probe connected to an oil manometer. The bulk flowrate is measured with a rotameter. All measurements in this exercise are analog and no calibrations are needed, though the Pitot measurements can be used to verify the accuracy of the rotameter. Students are required to study the radial and centerline velocity profiles of the jet at multiple flowrates, which they are given the liberty to choose.

\section{Description of the Lab Practical}

The lab practical (Lab 3b) was designed as a diagnostic exercise where TAs could observe students conducting a measurement to assess their experimental skills and teamwork habits, especially the combined effects of their development of physical intuition and live troubleshooting skills. In this exercise, the equipment was set up so that it was not working properly. Students were not told of this manipulation as the task was deigned to see if they were able to diagnose the error. No students were given a recipe of experimental procedures for the lab practical.

Students were expected to use a Pitot-static probe to measure the 2D velocity profile of air flowing through a square tube. The Pitot-static tube was connected to a Setra digital manometer with a full-scale of $125 \mathrm{~mm}$ of water, which was confirmed to be working correctly, and flexible clear plastic tubing was used to connect the Pitot-static tube to it through plastic barbed fittings. The equipment was modified by cutting part of the barbs off such that they would not hold pressure. Upon close inspection, it was evident that the barbs were not sealing against the tube. If the students found the problem with the barbed connection, the TA was instructed to give them a replacement barb so that they could proceed to fix the equipment and get more accurate measurements.

The proper dynamic pressure, if measured correctly, should have been approximately $55 \mathrm{~mm}$ of water, which corresponds to an air velocity of roughly $30 \mathrm{~m} / \mathrm{s}$. With the leaky connections, the errant dynamic pressure and velocity measured about $6 \mathrm{~mm}$ of water and $10 \mathrm{~m} / \mathrm{s}$, respectively. Thus, their digital measurement device appeared in every way to be working properly, but if the students took the effort to convert their pressure measurement to a velocity measurement, they may realize that the velocity they were measuring was too low, which admittedly may require some amount of intuition that the students probably do not yet have.

Because the exercise involves questions of accuracy, the students were also asked to assess the uncertainty in their measurements. Because the fluid density is needed to convert the dynamic 
pressure measurement into a velocity measurement, students are given the option to either assume an air density of $1.2 \mathrm{~kg} / \mathrm{m}^{3}$ at $5 \%$ uncertainty, or to use a mercury barometer and mercury thermometer to find a precise measurement of the air density, with an accuracy on the order of $1 \%$ or less. Whether teams actually took the time to measure these quantities is itself a matter of interest.

\section{Procedure}

One hundred twelve students, most of whom are Mechanical Engineering majors, enrolled in a junior-level introductory fluid mechanics laboratory course in the fall semester at the University of Illinois at Urbana-Champaign. Of them, one hundred four students signed a consent form to allow the use of their grades in this study. Students were not instructed not to talk about what they did in their lab sessions, whatsoever. They were not told anything about the study, other than their consent being requested. The TAs were instructed to say nothing about the different manners (recipe/no recipe) in which the lab was conducted on different days of the week. TAs were simply instructed to give recipes to $\mathrm{M} / \mathrm{W} / \mathrm{F}$ students nonchalantly, as though it is the normal way that the lab is conducted, without saying anything to draw unusual attention from their students. If students asked why they were given recipes, the TA would respond that it was to ensure that they followed a pre-tested procedure, and that the design of experiment that they had just completed was done as an academic exercise. Other than students signing consent forms early in the semester, there was nothing communicated to the students about whether they were participating in a study, the nature of the study, or when and how data was being collected. After the study was completed we noticed that one TA, who taught under both conditions, had deviated from the rubric while grading. The scores from their sections were not included in the analyses. After excluding these students, 87 students were analyzed. All students completed the labs described above. Grading of lab reports was done by the TAs through use of spreadsheet-based grading rubrics such that all points earned and all penalties applied were itemized and could be used for later analysis. A sample section of one of the grading rubrics is shown in Figure 2. 


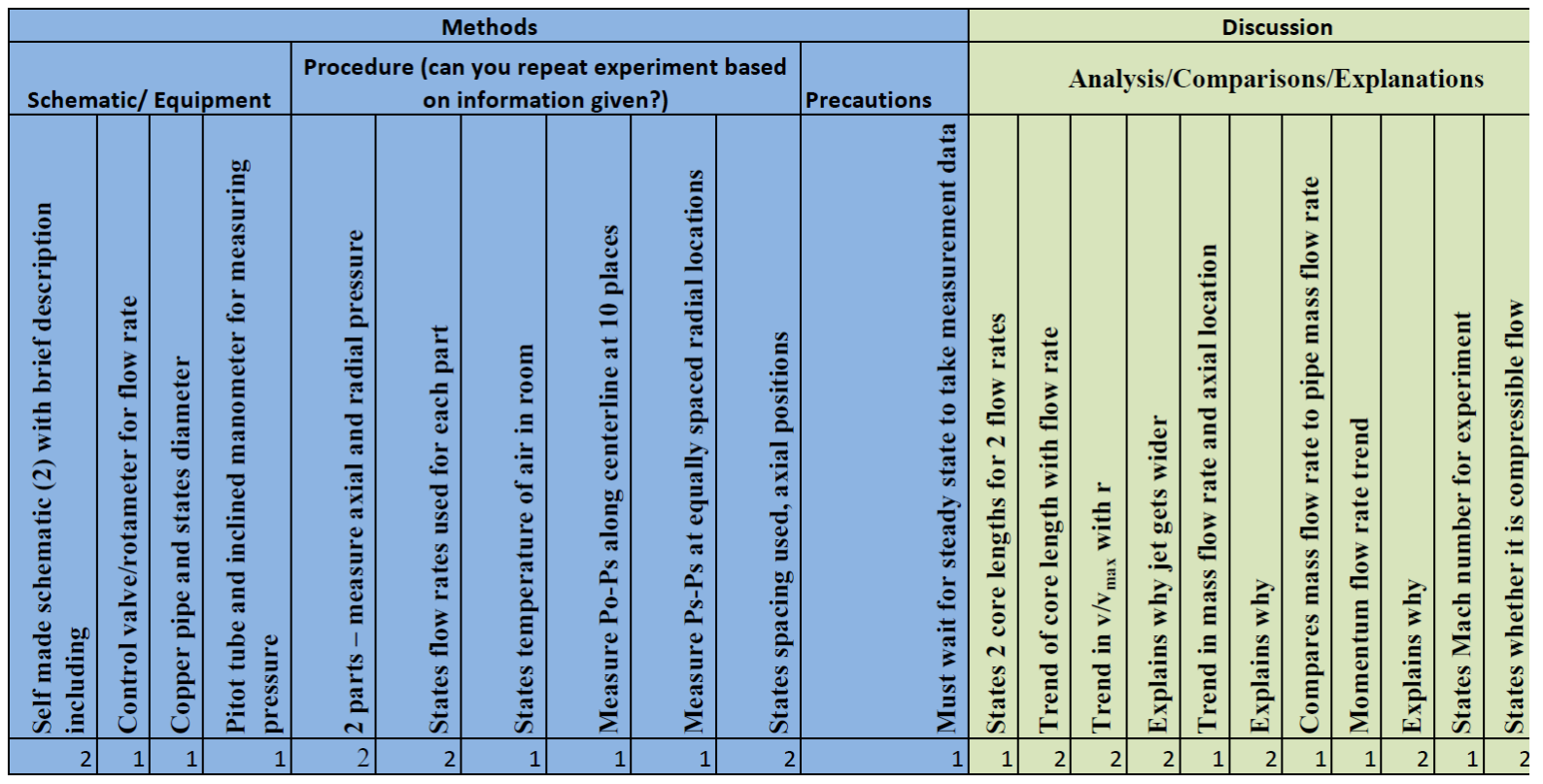

Figure 2. Portion of a spreadsheet-based grading rubric used by TAs to grade reports. The row of numerical values indicates the maximum points that can be earned for each item.

\section{Analysis and Results}

Table 3 contains a comparison of the scores on all three lab reports for the two conditions. The lab report scores were analyzed and were found be non-normally distributed, therefore nonparametric tests were used to analyze the differences in lab report score between conditions. To determine whether the absence of a recipe influenced the student's scores on their lab reports, a Mann-Whitney U-test was conducted. A significant difference between the conditions was found on all three of the lab reports. For Lab 2 a significant difference between the conditions was found $(z=3.21, p<0.01)$. The effect size $(d=0.71)$ indicates that this represents a large effect. For Lab 3 a significant difference between the conditions was found $(z=2.10, p<0.05)$. The effect size $(d=0.29)$ indicates that this represents a medium effect. For Lab $3 b$ a significant difference between the conditions was found $(z=2.89, p<0.01)$. The effect size $(d=0.58)$ indicates that this represents a medium effect. This indicates that students who did not receive a recipe received higher scores on their lab reports than students who did receive the recipe (see Figure 3). 
Table 3. Mean and standard deviation $(S D)$ for lab report scores by condition

\begin{tabular}{lcccc}
\hline & \multicolumn{2}{c}{ With Recipe } & \multicolumn{2}{c}{ Without Recipe } \\
\cline { 2 - 5 } Lab & Mean & $S D$ & Mean & $S D$ \\
\hline 2 & 75.5 & 12.4 & 82.8 & 7.7 \\
3 & 79.2 & 9.4 & 82.4 & 11.1 \\
$3 \mathrm{~b}$ & 88.7 & 5.2 & 91.2 & 3.1 \\
\hline
\end{tabular}

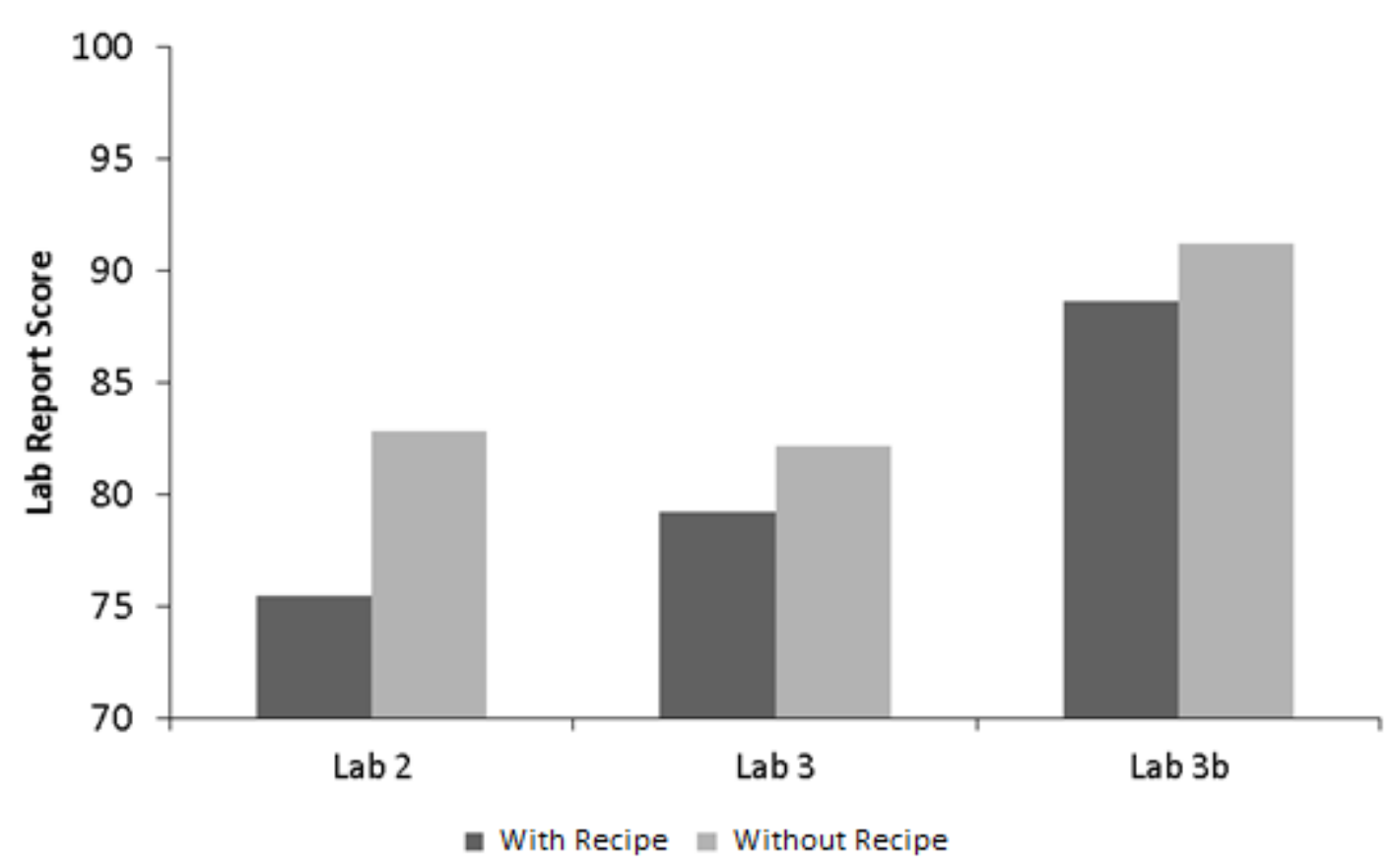

Figure 3: Lab Report Scores by Condition

To determine whether the absence of a recipe influenced the student's behavior in the labs, the TAs ratings for the lab practical were analyzed. TAs rated the student groups on a yes/no basis for the following criteria: Whether they arrived prepared to start quickly, for example, having thoroughly reviewed the laboratory manual; whether they used all of the allotted time to achieve highly-refined data. No students in either condition measured velocities on the order of $30 \mathrm{~m} / \mathrm{s}$, nor detected the faulty connection in the equipment, suggesting that they did not have intuition about how an airstream of a certain velocity should feel. On the other hand, all of the students in both conditions were effective in organizing their lab team and distributing the work across teammates. There were differences between the conditions for other behaviors. Table 4 contains the frequency of each behavior by the condition. Chi-square tests of independence were conducted to determine whether there were differences in the frequency of the behaviors between the conditions (see Table 4). The chi-square tests indicate that students who did not receive a recipe tended to come to the lab prepared and use all of their time more frequently than students who did 
receive the recipe, however the differences for behaviors related to making measurements in the lab were not significant.

Table 4. Means and $S D$ for the lab reports by condition

\begin{tabular}{lccccc}
\hline & \multicolumn{2}{c}{ With Recipe } & \multicolumn{2}{c}{ Without Recipe } & $\chi^{2}(1)$ \\
\cline { 2 - 6 } Lab & $n$ & $\%$ & $n$ & $\%$ \\
\hline Students came prepared & 24 & 68.6 & 54 & 100.0 & $19.4^{* *}$ \\
Students used all of their time & 32 & 91.4 & 54 & 100.0 & $4.8^{*}$ \\
Students used barometer and & 4 & 11.4 & 10 & 19.0 & 0.8 \\
thermometer to determine air density & & & & & \\
Team measured errant velocities & 25 & 71.4 & 41 & 76.0 & 0.2 \\
Team live-troubleshot their data & 22 & 62.9 & 32 & 59.0 & 0.1 \\
\hline$* p<.05, * * p<.01$. & & & & &
\end{tabular}

\section{Discussion}

We observe that a minor adjustment of the laboratory instruction in the direction of a guided inquiry environment yielded what appears to be a very positive outcome in terms of the student experience in the lab and, by extension, the overall quality of the lab course. This change seems to facilitate them to secure better grades and to participate actively in the collaborative laboratory exercises. Grade improvements of at least $2 \%$ in the mean were observed among those students who were not given recipes to follow. Behavioral differences were also noted in that students who were not expecting to benefit from being given a recipe proved much more likely to both come to lab prepared and to use all of the time allotted. Whether they used all of their time in this exercise is an indicator of their likelihood to accept data of moderate quality rather than of high quality. The exercise was to make velocity measurements over a $2 \mathrm{D}$ domain. The students were advised to start with a coarse grid of measurement points, such as $3 \times 3$, and to take additional data points to refine their grid as time allowed. Their choice was ultimately to decide whether it was in their best interests to use all of their time to achieve the highest spatial resolution possible. It was not indicated to them whether their grade depended upon the quality of their measurements.

The lab practical portion of the study contains significant room for improvement as a diagnostic tool for live troubleshooting habits. It seems that the fault in the equipment may be far too easy to overlook. A new approach where the students are required to connect the tubing themselves rather than having it connected in advance might be adopted, which would give students a better opportunity to observe to the poor condition of the equipment. Additionally, because it is clear that students do not have the physical intuition to tell whether $10 \mathrm{~m} / \mathrm{s}$ is too low of a velocity, students may be given additional information, such as the expected volumetric flowrate, which 
they may use to deduce the approximate velocity that should be expected. Another item that we had hoped would indicate a difference in with- vs. without-recipe student behavior was whether they used the mercury barometer and thermometer to make a precise and accurate measurement of the air density in the room. The lab manual suggested that they could simply use a value of $1.15 \mathrm{~kg} / \mathrm{m}^{3}$ with an assumed uncertainty of about $5 \%$ or that they could use the barometer and thermometer to make a precise measurement of the density. However, a very small number of students took the option to improve their measurements in this manner. It could be the case that the students simply accept the values presented to them to use as a result of the academic context of the laboratory scenario - a "why would they give me that value if I was not supposed to use it" mentality.

Part of the initial motivation for running the lab practical exercise had to do with the inherent trust that the students seem to have of digital measurement devices. We wonder to what degree experimental accuracy, precision, trustworthiness, and ease-of-use of the measurement devices affects the students' choice of what device to use. We also wonder whether the level of inquirybased instruction in the lab leads to students understanding and caring about the importance of making high quality measurements.

As this study continues, a deeper investigation is warranted. A more detailed analysis of student behavior when controlled for each TA can be performed since multiple TAs led lab session where recipes were and were not provided to the students. Video was taken of the students in at least three lab sections, which has yet to be analyzed to determine whether students who used the recipes participated more effectively with their teammates or not.

\section{References}

$1 \quad$ Froyd, Jeffrey E., Phillip C. Wankat, and Karl A. Smith. "Five major shifts in 100 years of engineering education." Proceedings of the IEEE 100.Special Centennial Issue (2012): 1344-1360.

2 Self, Brian P., and James Widmann. "Learning fundamental mechanics relationships using inquiry-based learning activities." Frontiers in Education Conference (FIE), 2014 IEEE. IEEE, 2014.

3 Regueras, Luisa M., et al. "Design of a competitive and collaborative learning strategy in a communication networks course." Education, IEEE Transactions on 54.2 (2011): 302-307.

4 Garrison, Thomas F., Michael R. Kessler, and Eric Hall. "A case study of the effects of a resident scientist on teaching experimental design to high school chemistry students." Interactive Collaborative Learning (ICL), 2014 International Conference on. IEEE, 2014.

5 Heer, Donald, Roger Traylor, and Terri S. Fiez. "TEKBOTS TM: Creating excitement for engineering through community, innovation and troubleshooting." Frontiers in Education, 2002. FIE 2002. 32nd Annual. Vol. 2. IEEE, 2002.

6 Buffler, Andy, Fred Lubben, and Bashirah Ibrahim. "The relationship between students' views of the nature of science and their views of the nature of scientific measurement." International Journal of Science Education 31.9 (2009): 1137-1156. 